\title{
Experimental Exploration of Decision Making in Production-inventory System
}

\author{
Felicjan Rydzak ${ }^{1}$, Agata Sawicka ${ }^{2}$ \\ 1 Centre for Advanced Manufacturing Technologies, Wroclaw University \\ of Technology, ul. Lukasiewicza 5, 50-371 Wroclaw, Poland \\ Phone: +48 713204184 / Fax: +48 713280670 \\ 2 Faculty of Science and Engineering, Agder University College \\ NO-4876 Grimstad, Norway \\ Phone: + 4737253358 / Fax: + 4737253001
}

\begin{abstract}
Despite availability of advanced decision aids, manufacturing managers should master basic control tasks within production systems. Still, there is substantial evidence that even highly educated and experienced practitioners mismanage even very simple production-inventory systems. We report an experimental study investigating strategies in a simple productioninventory control task. The study was conducted using an interactive computer simulator. Consistent with previous observations, the subjects failed to execute a satisfactory control in the first trial. Most, however, learned to manage the system as they gained experience. Application of single subject experimental design with think-aloud protocol and debriefing interviews allowed to identify strategies followed by the subjects. The paper reports on the key results of the study, discussing its implications for the production management research and practice.
\end{abstract}

\section{Introduction}

Production-inventory control is fundamental for effective management of both manufacturing process and manufacturing supply chain. Regardless of the complexity of the relationships within the production-supply environment, the main challenge is unchanged - i.e., to have the right number of right products at the right place and time. This is difficult because of the inherent delays in the flow of materials, information and money, as well as ever changing and unpredictable demand $[1,2]$.

Over the last two decades various strategies and approaches have been proposed to improve decision making along a whole supply chain as well as at a particular echelon. One line of research advocates development of robust control algorithms to optimize the use of available information and consequently improve the inventory

Please use the following format when citing this chapter:

Rydzak, F. and Sawicka, A., 2008, in IFIP International Federation for Information Processing, Volume 257, Lean Business Systems and Beyond, Tomasz Koch, ed.; (Boston: Springer), pp. 205-213. 
management. Towill [1] and White [3], for instance, applied control theory and more sophisticated control algorithms, such as proportional, derivative and integral (PID), to reduce inventory level by $80 \%$ - a result especially desired in just-in-time (JIT) or material requirements planning (MRP) techniques. The latest research investigates possibility of fuzzy logic control application [4]. However, better control algorithms are very often too sophisticated to be successfully incorporated into managerial practice.

Another line of research focuses on decision making processes in productionsupply systems. Production management games have been developed - with a pioneering Beer Game created by Jay W. Forrester at MIT in the beginning of $1960 \mathrm{~s}$ - to create virtual production management laboratories. These laboratories have been used often as training aids $[5,6]$. However, they have also been applied in research. Experimental results show that most people fail to control even overtly simplistic systems [7-11]. Forrester [12] and Sterman [8] suggest that people instead of developing a good understanding of the system rely on an anchoring and adjustment heuristic - a rule that starts with a salient reference point (anchor) and adjusts it to reach a final value; the problems occur because the adjustments are insufficient.

This paper reports on findings from an experimental study on decision-making in a simulation-based environment called INVENT. The study investigated strategies applied in a production-inventory control task. Consistent with previous findings [7, 10], we observed that people have difficulties in controlling a very simplified production-inventory system - one echelon of the whole supply chain. While most subjects initially used an erroneous anchoring and adjustment heuristic, many successfully improved the rule after a period of hands-on experience in managing the system. In decade when companies compete through their supply chains [13] improving people's ability to effectively manage production-inventory systems becomes increasingly important. Our results suggest that applications like INVENT may be a valuable aid in various training programmes for both students and practitioners of industrial management.

The paper is organized as follows: The next section discusses the design and methodology of the experiment. Following, we present the key findings. Finally, conclusions are drawn indicating limitations, implications and contributions of the research.

\section{Design and methodology}

The experimental study was conducted using a simulation-based production game called INVENT. The game was developed based on a System Dynamics model [2]. The structure of the model is presented in Fig. 1. The model consists of three sectors: Production and Inventory, Customer Orders, and Costs. In the System Dynamics notation rectangle boxes represent accumulations within the system - in our case they are stocks of elements in production (Production in Progress), stock of ready products (Inventory) and costs incurred during a period of an experiment (Cumulative Costs). Values of the accumulations within the system are changed by flows (arrows with valves) representing the movement of materials, information and 
cost; for instance, Planned Production indicates information impulse that starts production process, Shipment Rate indicates shipment of finished products to the customers. Additional variables specify certain parameters of the model, e.g. production lead time (Manufacturing Time) equal to 4 weeks. Usually System Dynamics models include also decision functions specifying the way in which the material flows are controlled. However, in this case that part of the model was replaced by the direct decisions of the experiment subjects.

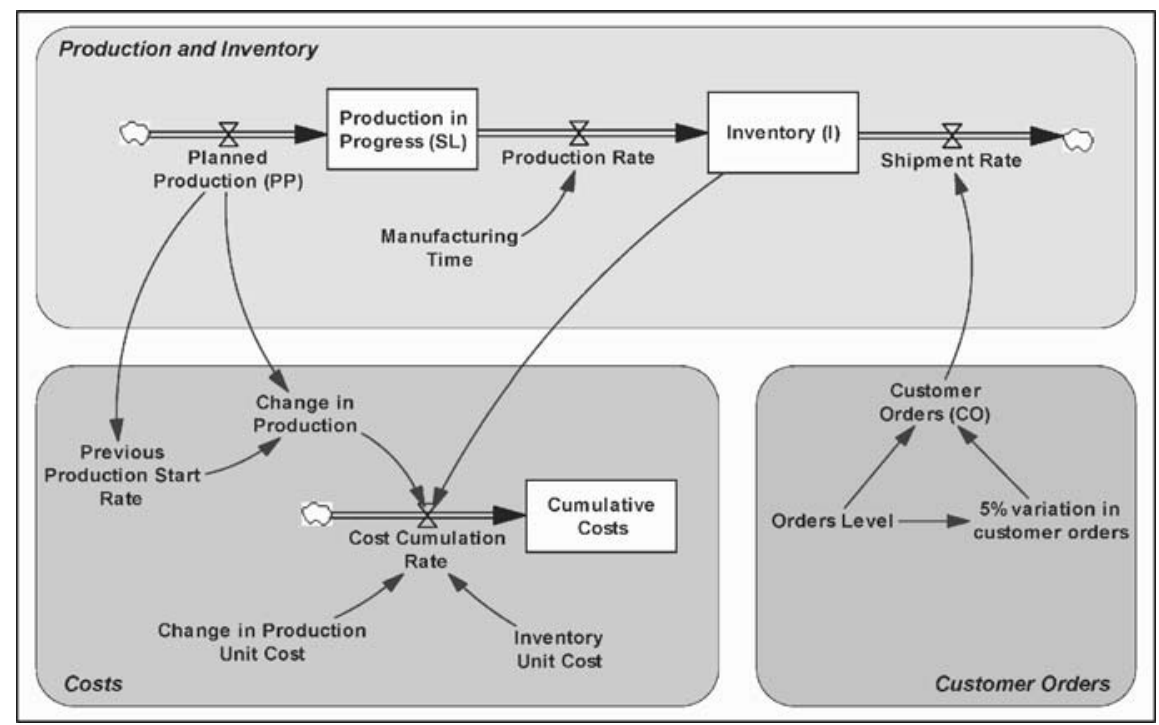

Fig. 1. The System Dynamics model structure of a simple production-inventory system used in the interactive learning environment INVENT

For the INVENT game, four different decision-making interfaces were designed. All four interfaces contained a spreadsheet like report on the problem variables and provided complete information about the current and past results. They differed in how explicitly they exposed the inherent manufacturing delay. The example shown in Fig. 2 presented the delay in the most explicit way by providing an animated diagram of the production-inventory system.

The study was designed as a single subject experiment [14] and involved 15 students attending MSc course in Management and Manufacturing Engineering at Wroclaw University of Technology. Because of the applied design, each subject attended the experiment individually.

The subjects took on the role of a production manager of one of the divisions of the ABC Manufacturing Co. At the start of the experiment each subject read the instructions. The subjects were asked to set weekly production so to minimize costs of operations. To accomplish this goal the inventory level should be maintained at the minimal level ('zero inventory') but simultaneously order losses should be avoided. Subjects were informed of the starting value of customer order and that they 
should expect the orders to follow a random walk with $+/-5 \%$ fluctuations and to have a one-time, step increase within the first 10 of the 32 simulated weeks.

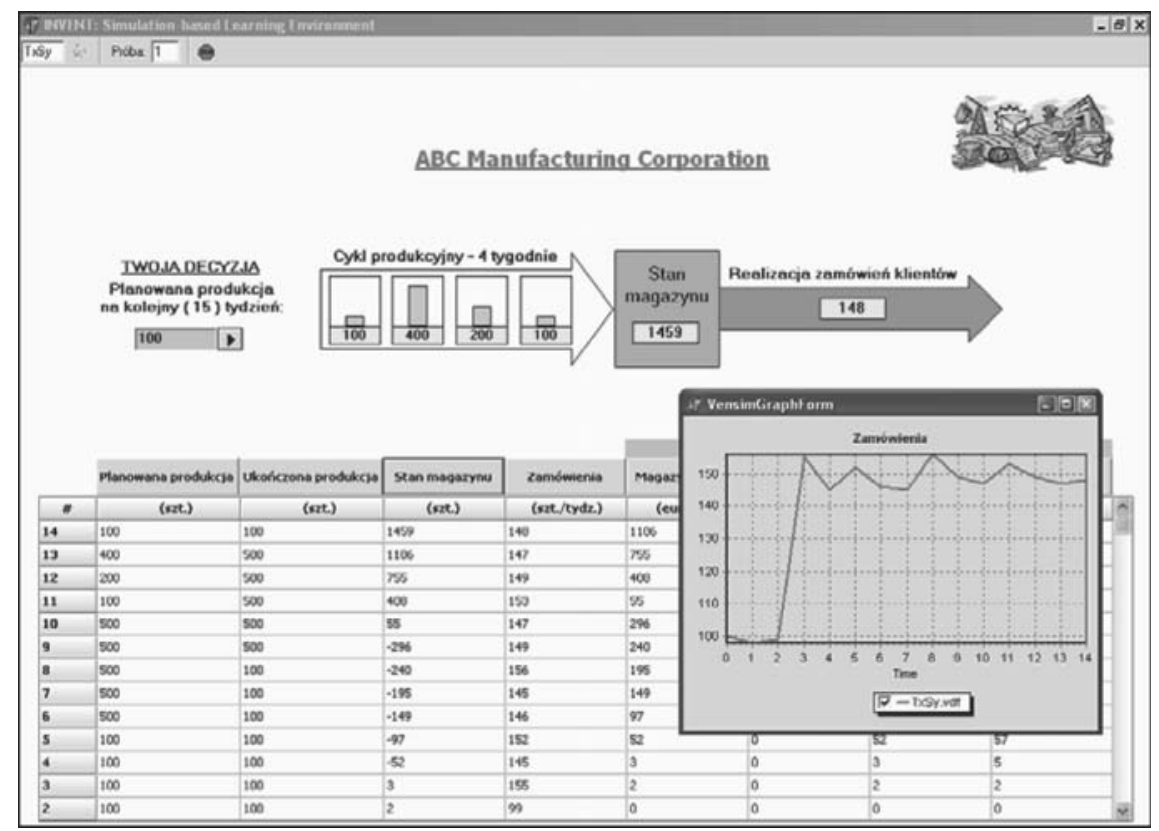

Fig. 2. The example of the interactive learning environment INVENT interface

Before playing INVENT subjects filled in a prior-test knowledge questionnaire. Next, they played INVENT three times. Each decision and all results were saved in a computer log. During conducting the task the subjects were encouraged to comment on their decisions ('think-aloud protocol'). After each trial an interview was conducted to elicit self-evaluation of the performance. At the end of the experiment each subject filled in the post-test questionnaire and evaluated the whole task.

\section{Findings}

The anchoring and adjustment heuristic proposed by Sterman [8] assumes that, when controlling inventories, the subjects anchor their decisions (Ot) in the imminent expected losses (ExpLt) and adjust for the inventory (I) and supply line (SL) discrepancies:

$$
O_{t}=M A X\left[0, \operatorname{Exp}_{t}+\left(I^{*}-I_{t}\right)+\left(S L^{*}-S L_{t}\right)\right]
$$

where $t$ indicates the current point in time and $*$ indicates the desired values.

Based on regression analysis, Sterman suggests that poor inventory control may be attributed to the subjects' fixation on the initial inventory level and their failure to account fully for the supply line [8, see pp.334-335]. 
To evaluate performance of our subjects, we compared their results with Sterman's anchoring and adjustment rule. Table 1 presents an overview of the subjects' inventories together with a reference inventory achieved when the anchoring and adjustment heuristic (1) is followed correctly. In Sterman's experiment, mismanagement following the demand increase led to inventory fluctuations [8, see Fig. 4, p. 330]. In our experiment we also observe such fluctuations, especially in the 1st trial (see Table 1). However, to determine whether the subjects indeed misapplied the anchoring and adjustment heuristic proposed by Sterman other data need to be also examined.

Table 1. Inventory levels - overview of the experimental results. Grey, bold line tracks the inventory level simulated using Steraman's anchoring and adjustment heuristic. Black colour is used to mark inventories of the individual subjects

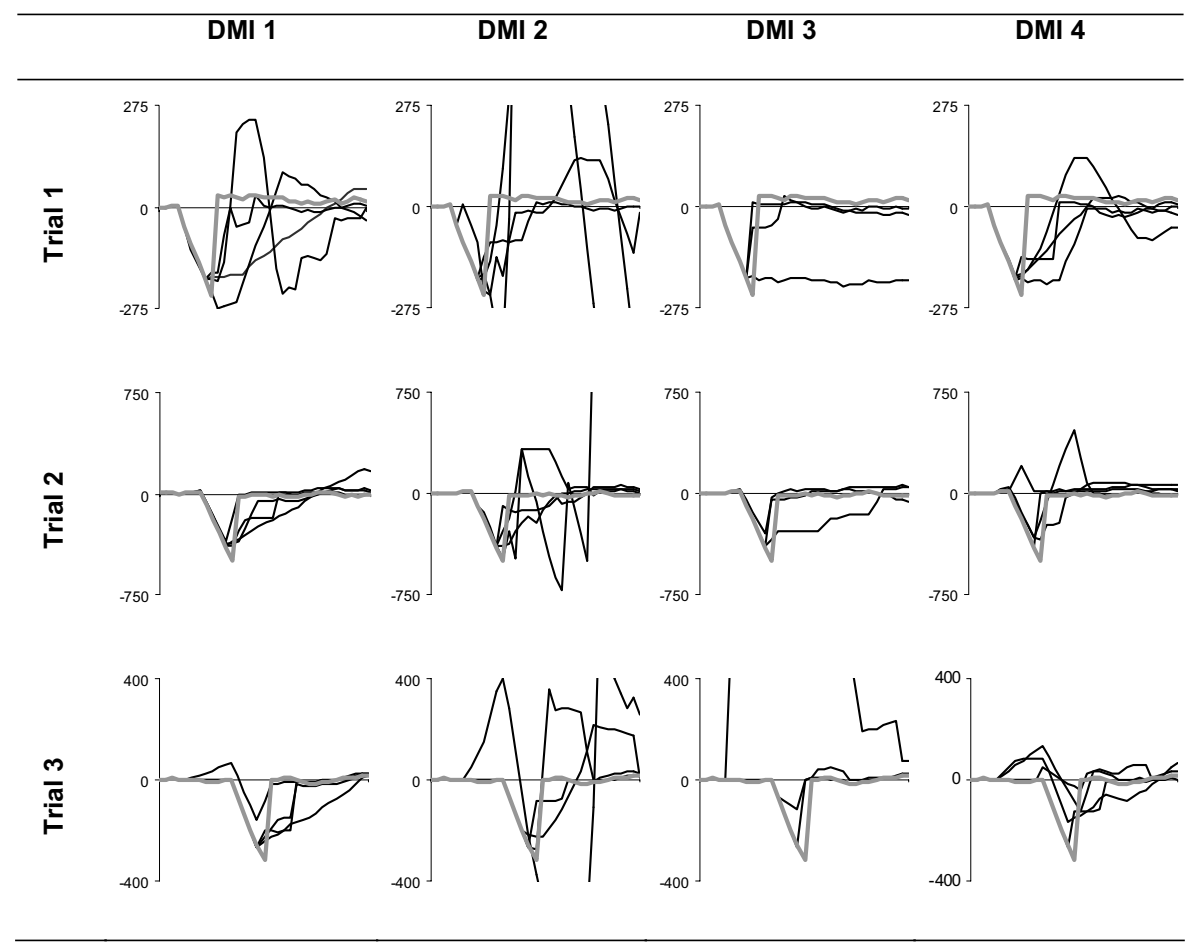

When comparing the cumulative costs, many of our subjects outperformed the heuristic - their cumulative costs were lower than those generated when the heuristic-based decisions were simulated. Further analysis of the think-aloud protocols suggested that most successful subjects did not follow the anchoring and adjustment heuristic as proposed by Sterman [8].

During the first trial only one subject controlled the system completely all the time. His decision rule was anchored in the imminent expected losses (represented 
by the current customer order level, CO) but it did not involve explicit adjustments for inventory or supply line. The subjects adjusted rather for the losses expected over the next 4 weeks (i.e., duration of the manufacturing delay) and the rule applied may be formulated as follows:

$$
O_{t}=M A X\left[0, C O_{t}+\sum_{i=1 . .4}\left(\operatorname{Exp} C O_{i}-P P_{i}\right)\right]
$$

where ExpCO indicates the expected level of customer orders and PP indicates planned production.

Most subjects ( 9 out of 10 ), who managed to stabilize the inventory over the three trials, developed a similar decision rule. 1

While the anchoring and adjustment heuristic proposed by Sterman [8] (see equation (1)) is robust for any type of customer order fluctuations, the decision rule followed by our subjects (see equation (2)) is robust only for the one-time step increase in the customer orders. As such, it could be deemed as suboptimal. However, given that the subjects were asked to respond to a specific customer order change, it is plausible to accept the rule as appropriate.

The rule developed by our subjects is arguably easier to apply than Sterman's anchoring and adjustment heuristic. When the decision making interface does not provide information on supply line explicitly - as it was in the original study by Sterman [8] or other similar experiments (see e.g., $[9,10,15]$ ) - its estimation is not straightforward. To facilitate this exercise in our study we provided visual hints on supply line volume. ${ }^{2}$ However, even the subjects that used these extended interfaces resorted to the rule that does not explicitly incorporate the supply line (see equation (2)).

The experiment did not reveal any significant influence of the decision making interfaces on the performance. Majority of the subjects (14 out of 15) in the first trial were surprised and confused by the effects the manufacturing delay had on their inventories. The results of the pre-task questionnaire indicated that most subjects understood the task well and only 2 subjects failed to provide a correct answer to the question about the manufacturing delay. Still, analysis of the think-aloud protocols and the debriefing interviews conducted after the first trial revealed that a number of subjects did not know how to act upon this information (4 out of 15) or have forgotten about it altogether ( 3 out of 15). In the post-task interviews roughly a half of the subjects (6 out of 15) indicated they would have wished to go through a training session before the start of the "real" game or to have a worked-out example illustrating how the delay works. In case of all 9 subjects who were eventually successful, it was clear that their performance improved as they gained experience. These observations suggest that provision of all necessary data alone is not likely to be sufficient for people to control effectively a production-inventory system. A hands-on-experience has a vital role in building the true understanding of the system and in developing the ability to control it.

\footnotetext{
${ }^{1}$ The one subject who controlled INVENT successfully while not following the described decision rule misunderstood the instructions, and tried to achieve a zero inventory level only at the end of the trial rather than as soon as possible.

${ }^{2}$ The most explicit presentation of the supply line was the interface presented in Fig. 2.
} 


\section{Conclusion}

The System Dynamics model used in the experiment focused mainly on the dynamics resulting from production delay. It considered only one echelon of a supply chain and did not include such constraints as production capacity or raw materials availability. To facilitate development of decision rules, the applied model of costs assumed equal unit costs of inventory and change in production. Despite such simplified environment, we managed to observe problems common to more complex supply chain systems. Since any simplification of the experimental environment facilitates analysis of decision making processes, this finding is positive $[16,17]$.

Rather than group design, we used a single subjects approach collecting only a small number of observations. Consequently, we cannot make any generalizations based on our results. However, we were able to gain a unique insight into individual decision making, unattainable in the group design. The value of such deeper insight is illustrated well by our observation that although the subjects' performance trails are similar to those observed earlier, our data suggest that the subjects relied on different anchoring and adjustment heuristic than this defined by Sterman [8]. This suggests that the single-subject design may be more suitable for investigations aiming to uncover decision rules.

In the current study we have not managed to observe any clear effects of different decision making interfaces on the subjects' performance. This may be due to the limited number of observations we gathered per interface - each interface type was used by only 3 to 4 subjects. Further studies are necessary to explore whether there are certain elements of the decision making interface that may facilitate the production-inventory task.

Still, even with the current, imperfect decision-making interfaces, we observed that many of our subjects developed successful decision rules in the course of the three trials. These results suggest that a hands-on experience is important for learning successful management of inventory-production systems. Indeed, many subjects emphasized that they had fully understood the task and the system only after a period of practice. Hence, it seems advisable to advocate for INVENT-like games to become an integral part of the manufacturing training programmes both at the university and professional levels.

\section{Acknowledgements}

The study reported in this paper has been initiated by Dr. Agata Sawicka as a part of a greater research project sponsored by the Research Council of Norway (grant no. 160789/V30). It has been carried out at two research institutions - Agder University College, Norway, and Technical University of Wroclaw, Poland; support of the research leaders at both institutions Professor Jose J. Gonzalez and Professor Edward Chlebus, respectively, is gratefully acknowledged. We also thank Dr. Piotr Magnuszewski and Piotr Obrapalski, MSc, for their help in development of the INVENT simulation environment. 


\section{Reference:}

1. Towill, D.R., Dynamic analysis of an inventory and order based production control system. International Journal of Production Research, 1982. 20(6): p. 671687.

2. Sterman, J.D., Business Dynamics. Systems thinking and modeling for a complex world. 2000: McGraw-Hill.

3. White, A.S., Management of inventory using control theory. International Journal of Technology Management, 1999. 17(7/8): p. 847-860.

4. Ge, Y., et al. Controller Design for the System Dynamics Model of a UK Supermarket Supply Chain. in 6th World Multi-conference on Systemics, Cybernetics and Informatics. 2002. Orlando, USA.

5. Rauch-Geelhaar, C., K. Jenke, and C.M. Thurnes, Gaming in industrial management - quality and competence in advanced training. Production Planning \& Control, 2003. 14(2): p. 155.

6. Hofstede, G.J., et al., A chain game for distributed trading and negotiation. Production Planning \& Control, 2003. 14(2): p. 111.

7. Sterman, J.D., Testing behavioral simulation models by direct experiment. Management Science, 1987. 33(2): p. 1572-1592.

8. Sterman, J.D., Modeling managerial behavior: Misperceptions of feedback in a dynamic decisin making experiment. Management Science, 1989. 35(3): p. 321.

9. Croson, R. and K. Donohue, Behavioral Causes of the Bullwhip Effect and the Observed Value of Inventory Information. Management Science, 2006. 52(3): p. 323336.

10. Diehl, E. and J.D. Sterman, Effects of Feedback Complexity on Dynamic Decision Making. Organizational Behavior and Human Decision Processes, 1995. 62(2): p. 198-215.

11. Hieber, R. and I. Hartel, Impacts of SCM order strategies evaluated by simulation-based 'Beer Game' approach: the model, concept, and initial experiences. Production Planning \& Control, 2003. 14(2): p. 122.

12. Forrester, J.W., Industrial Dynamics. 1961, Cambridge, MA: The M.I.T. Press.

13. Hill, T., Manufacturing Strategy - Text and Cases. 2 ed. 2000, Houndsmills, Hampshire: Palgrave.

14. Barlow, D.H. and M. Hersen, Single Case Experimental Designs: Strategies for Studying Behavior Change. 2nd ed. 1984, New York: Pergamon Press. 
15. Wu, D.Y. and E. Katok, Learning, communication, and the bullwhip effect. Journal of Operations Management, in press.

16. Edwards, W., Conservatism in human information processing, in Judgment under Uncertainty: Heuristics and Biases, D. Kahneman, P. Slovic, and A. Tversky, Editors. 1982, Cambridge University Press: Cambridge, UK.

17. Moxnes, E., Misperceptions of basic dynamics: the case of renewable resource management. System Dynamics Review, 2004. 20: p. 139-162. 